\title{
Inherited Proximal Tubular Disorders and Nephrolithiasis
}

Ben Oliveira $^{1}$, Robert Unwin ${ }^{1}$, Stephen B Walsh ${ }^{1}$

1. UCL Centre for Nephrology, London, UK

Correspondence to:

Stephen B Walsh

Senior Lecturer in Experimental Medicine and Honorary Consultant Nephrologist

Centre for Nephrology University College London

Royal Free Hospital / Medical School

London NW3 2PF, United Kingdom

Email: ucgbsbw@ucl.ac.uk 


\section{Introduction}

Classically, the distal tubule is often linked to nephrolithiasis; however, several inherited proximal tubular disorders have also been associated with renal stone disease. While these conditions might be rare, they provide clues as to how the proximal tubule handles solutes involved in stone formation, and can also provide insights into the pathogenesis of more common forms of stone disease.

\section{Overall Functions of the Proximal Tubule}

The proximal tubule is the workhorse of the human nephron responsible for claiming back the majority of the $160-170$ litres of glomerular filtrate produced every day. Specifically $60-70 \%$ of filtered $\mathrm{NaCl}$ and water, and all of the $\mathrm{NaHCO}_{3}$ is reabsorbed in this nephron segment ${ }^{1}$. It is also the site of reabsorption for glucose, amino acids and several important anions (including phosphate and citrate). The proximal tubule also has a metabolic function. It is responsible for both the 1-alpha hydroxylation of vitamin $D$ to its active form and the inactivation of this hormone via the 24-hydroxylase reaction ${ }^{2}$. Of the solutes reclaimed in the proximal tubule, phosphate is the one of the most important in relation to risk of nephrolithiasis. 


\section{Phosphate \& Calcium Homeostasis in the Proximal Tubule}

The proximal tubule is responsible for reclaiming about $80 \%$ of the filtered phosphate under normal physiological conditions. Two sodium dependent phosphate cotransporters of the SCL34 solute carrier gene family carry out most of this ${ }^{3}$. The bulk (85\%) is carried out by SLC34A1 (NaPi-Ila). SLC34A3 (NaPi-IIc) reabsorbs the remaining $15 \%{ }^{3}$. SCL34A2, another member of this gene family, is mainly expressed in the small intestine.

Another family of sodium dependent phosphate transporters, designated SLC20, is also expressed in the proximal tubule; however, the overall contribution of these transporters to phosphate reclamation is likely to be small ${ }^{4}$. The entry of phosphate through these cotransporters is driven by the cotransport of sodium, the electrochemical gradient of which is established by the sodium/potassium ATPase located on the basolateral cell membrane ${ }^{5}$.

Expression of $\mathrm{NaPi}-\mathrm{Ila}$ is at least partly regulated by parathyroid hormone (PTH). PTH binding to G-protein coupled type 1 PTH receptors (PTH1R) activates protein kinase $\mathrm{A}(\mathrm{PKA})$ via cAMP, and protein kinase $\mathrm{C}$ $(\mathrm{PKC})^{6,7}$. Once activated, these kinases phosphorylate the PDZ domain of NHERF18. NHERF1 anchors NaPi-lla to the cytoskeleton, but releases it when phosphorylated, leaving NaPi-lla free to be endocytosed; see figure $1^{9}$. The action of PTH, therefore, is to internalize NaPi-Ila, leading to phosphate wasting $^{10,11}$. The other action of PTH in this segment is inhibition of the sodium proton exchanger isoform $3(\mathrm{NHE} 3)^{12}$. This exchanger extrudes protons into the tubular lumen in exchange for the reabsorption of sodium. The combination of hydrogen ions with filtered bicarbonate allows for the 
effective reclamation of bicarbonate through passive diffusion of carbon dioxide across the tubular membrane. This exchanger also sets up a concentration gradient for the reabsorption of water molecules ${ }^{13}$. So, inhibition of NHE3 will lead to deceased sodium and water reabsorption and alkalisation of the tubular fluid. A result of this is to decrease calcium reabsorption. There are two mechanisms for this: firstly, paracellular calcium uptake in the proximal tubule appears to be coupled to sodium reabsorption; indeed, mice lacking NHE3 have an increased fractional excretion of calcium, despite elevated levels of activated vitamin $D^{14,15,16}$. Secondly, the actions of NHE3 are to acidify the urine, this not only creates more ionised calcium for absorption in more distal segments, but also potentially increases the activity of the calcium transporter TRPV5. So, the PTH mediated inhibition of NHE3 acts to decrease calcium reabsorption in the proximal tubule, the opposite of its overall effect on calcium excretion in vivo. Stimulation of the calcium sensing receptor (CaSR) in this nephron segment opposes the actions of PTH; it abolishes PTH mediated internalization of NaPi-Ila and stimulates NHE3 ${ }^{17,18}$. Stimulation of CaSR, therefore leads to increased phosphate, sodium and water reabsorption, and acidification of tubular fluid. The net result of this is an increase in proximal tubular calcium reabsorption. It should be noted that the presence of CaSR in the proximal nephron is controversial. While mRNA and immunolocalization studies had demonstrated CaSR expression throughout the nephron ${ }^{19,20}$, Loupy and coworkers reported expression confined to the thick ascending $\operatorname{limb}(\mathrm{TAL})^{21}$. More recently, a comprehensive study has shown that although expression of the CaSR is highest in the TAL, there is expression elsewhere including the proximal 
tubule $^{22}$. Interestingly, CaSR expression in the proximal tubule and collecting duct is on the apical membrane while in the $\mathrm{TAL}$ it is basolateral ${ }^{20}$. It has been proposed that proximal tubular CaSR serves to mitigate overall calcium excretion and reduce the risk of calcium salt precipitation. The CaSR in the proximal nephron may therefore serve a role in protecting the kidney against stone formation.

Another important regulator of proximal tubular phosphate reabsorption is Fibroblast Growth Factor-23 (FGF-23). FGF-23 binding to the FGF1c receptors leads to down regulation of both $\mathrm{NaPi}-\mathrm{Ila}$ and $\mathrm{II}^{23}$. FGF-23 also leads to decreased levels of $1,25(\mathrm{OH})_{2} \mathrm{D}_{3}$ due to inhibition of one alpha hydroxylase activity²4.

\section{Phosphate \& Calcium Transport \& Nephrolithiasis}

\section{SLC34A1/NaPi-Ila}

SLC34A1 lies on chromosome $5 q 35$ and consists of thirteen exons and twelve introns. SLC34A1 transports three sodium ions together with one divalent phosphate ion ${ }^{25,26}$. Bi-allelic mutations in this gene have been reported in an Palestinian family with hypophosphataemic rickets ${ }^{27}$. More recently, homozygous mutations have been found in a family with Idiopathic Infantile Hypercalciuria $(\mathrm{IIH})$ that did not have a mutation in $C Y P 24 A 1^{28}$. Secondary increases in vitamin D and hypercalciuria were also seen, as well as renal tract calcification. Neither of the two Palestinian cases had nephrolithiasis; the authors speculated that this was probably due nutritional 
vitamin $\mathrm{D}$ deficiency. In the absence of a nutritional defect phosphate wasting should lead to increased activation of vitamin D, leading to increased intestinal calcium absorption and hypercalciuria. The patients also had a generalised renal Fanconi type defect, leading to citrate wasting; this may also have protected them against stone formation. The mutation in this family was an in-frame inserted duplication in SLC34A1 (p.I154 V160dup-NaPilla). The mutation leads to a trafficking defect and retention of the protein in the endoplasmic reticulum (ER). The same authors later speculated that disruption of the ER by the mutant protein could affect other proteins in the cell, explaining the generalised proximal tubulopathy ${ }^{27,29}$.

A candidate gene approach in individuals with Hypophosphataemic Nephrolithiasis with Osteoporosis revealed heterozygous mutations of $S L C 34 A 1^{30}$. The pathogenic significance of this finding is not clear; the mutation has not been found in other kindreds with the same phenotype, and expression of mutant SLC34A1 did not affect phosphate transport in cell models ${ }^{31}$.

Mutations in sodium-hydrogen exchanger regulator factor 1 (NHERF1) have also been found in individuals with Hypophosphataemic Nephrolithiaisis with Osteoporosis. NHERF1 binds to NaPi-Ila and PTH. PTH regulates phosphate by reducing NaPi-Ila expression at the apical membrane of proximal tubule cells. Members of NHERF family of proteins are proposed to scaffold various transporters at the apical membrane, preventing their internalisation into endosomes. The PDZ1 domain of NHERF1 is crucial for its interaction with $\mathrm{NaPi}-\mathrm{Ila}$ and phosphorylation of this domain decreases its affinity for $\mathrm{NaPi}$-lla, leaving it free to be internalised into endosomes. 
Activation of PTHR1 by PTH causes increased cAMP production, activating the protein kinase $A(P K A)$ pathway that ultimately leads to phosphorylation of the PDZ1 domain of NHERF1. NHERF1 is crucial for producing the phosphaturic effects of PTH. Indeed, the coupling of apical PTHR1 stimulation to down-regulation of NaPi-Ila expression is completely abolished in NHERF1 -/- mice ${ }^{32}$. NHERF1 also modulates the amount of cAMP produced by the PTHR1 receptor in response to PTH stimulation. This interaction occurs via the PDZ2 domain of NHERF1. Mutations of the PDZ2 domain abolish the interaction between NHERF1 and PTHR1, leading to unabated cAMP production in response to $\mathrm{PTH}$ and, therefore, an increased phosphaturic effect. Such mutations have been shown to produce phosphaturia and nephrolithiasis in humans ${ }^{33}$. Subsequently, a mutation has been described in the PDZ1 domain, leading to phosphaturia and nephrolithiasis ${ }^{34}$. As PDZ1 does not interact with PTHR1, this mutation limits NaPi-lla expression in a PTH independent fashion.

Variants in SLC34A1 have also been implicated in stone formers who do not have Mendelian disease. A Japanese genome wide association study (GWAS) of 5,892 patients with calcium-containing stones found three loci associated with stone formation, one of which occurred in intron four of SLC34A135. Although the authors found an association of this variant with reduced renal function, there was no significant association with serum phosphate. However, a previous GWAS did find an association of serum phosphate and variants at $S L C 34 A 1^{36}$. Furthermore, knockout-mice for NaPi-Ila develop phosphate wasting with secondary hypervitamin D production and hypercalciuria ${ }^{37}$. 


\section{SLC34A3/NaPi-Ilc}

SLC34A3 gene spans $5 \mathrm{~kb}$, contains thirteen exons and is located on chromosome nine. It transports two sodium ions for every divalent phosphate ion and is therefore electro-neutra| ${ }^{38,39}$. Hereditary Hypophosphataemic Rickets with Hypercalciuria $(\mathrm{HHRH})$ is an autosomal recessive condition presenting with bone disease, hypercalcaemia and nephrolithiasis ${ }^{40}$. Compound heterozygous mutations have been reported to cause the condition ${ }^{38}$. Individuals with just one mutated allele do not have the HHRH phenotype, but varying degrees of hyperphosphaturia and hypercalciuria have been reported in the literature ${ }^{41}$.

A linkage study performed in a Spanish kindred identified a locus spanning SLC34A3 on the long arm of chromosome nine associated with autosomal dominant inherited nephrolithiasis ${ }^{42}$. Another group has shown that in family members of HHRH sufferers, heterozygotes of $S L C 34 A 3$ mutations were more susceptible to kidney stones with a three-fold increase in risk compared with the general population ${ }^{43}$.

\section{CYP24A1}

$\mathrm{IIH}$ type 1 is caused by inactivating mutations in the CYP24A1 gene that encodes $25-\mathrm{OH}-\mathrm{D}_{1}-24-$ hydroxylase, the enzyme responsible for inactivating vitamin $D^{44}$. This was first recognized in the 1950s with children presenting with symptomatic hypercalciuria when given milk fortified with vitamin D. However, the same defect is also found in those presenting for the first time as adults with nephrocalcinosis and calcium phosphate nephrolithiasis ${ }^{45}$. 
Without CYP24A1, vitamin D continues to exert its effects, including increased calcium reabsorption, leading to hypercalcaemia and the development of nephrolithiasis

\section{Hypophosphataemic Rickets}

It is worth touching on this condition associated with phosphate wasting, but not with nephrolithiasis. The phosphate wasting is due to increased activity of FGF-23 that decreases the activity of both NaPi-lla and NaPillc. However, FGF-23 also leads to less active vitamin D and so hypercalciuria does not ensue. This highlights that hypophosphaturia alone is probably not enough to cause nephrolithiasis, which may be because it requires calcium to crystalize.

\section{CaSR}

The CaSR has a key role in regulating both calcium and phosphate excretion in the kidney. Variants in CASR are associated with nephrolithiasis ${ }^{46}$. Work by an Italian group has shown that the CASR polymorphism Arg990Gly was more common in hypercalciuric stone formers than controls. In vitro work revealed that this was a gain-of-function mutation ${ }^{47}$. However, other investigators failed to show any interaction with the SNP and calcium homeostasis $^{48}$. The SNP rs6776158 located in the first promoter of CASR has also been associated with calcium nephrolithiasis. Further work revealed that the minor G allele of this SNP caused decreased transcription in renal cell lines. Soldati's group suggested that the combination of rs6776158 and Arg990Gly polymorphisms potentiates the risk of nephrolithiasis ${ }^{49}$. This seems strange, given that these two SNPs appear to have opposite effects on 
CaSR expression. However, given the complex role that CaSR has in regulating calcium homeostasis in the kidney, it may well be that disrupting this regulation (either positive or negative) is enough to tip the balance in favour of calcium salt precipitation. Gain-of-function mutations can easily be imagined to cause nephrolithiasis, given that the actions of CaSR are to promote calcium wasting in the urine. Decreased expression of the receptor in certain areas of the nephron could also have detrimental consequences. For example, decreased expression in the proximal tubule would abolish the protective affects (i.e. calcium reabsorption) at this site, leading to more calcium delivery to the distal nephron. This speculative mechanism needs further work to clarify the exact effects these variants have on renal calcium handling.

\section{$\underline{\text { Renal Fanconi Syndrome and Other Transporters }}$}

The renal Fanconi syndrome describes generalised loss of proximal tubular function including wasting of bicarbonate, phosphate, glucose and amino acids. Children with Fanconi syndrome present with failure to thrive and rickets. A number of inherited disorders have been described that cause Fanconi syndrome. Despite phosphate wasting being a feature of many of these conditions, nephrolithiasis is really only a feature of Dent disease and Lowe syndrome.

\section{Dent Disease}


Dent and Friedman first described the condition in 1964 in two children with rickets, aminoaciduria, phosphaturia and hypercalciuria ${ }^{50}$. Further characterisation of the condition by Oliver Wrong identified both nephrocalcinosis and nephrolithiasis as features of this condition ${ }^{51}$. Several linkage studies in the 1990s mapped the causative gene to Xp11.22, confirming it as an X-linked condition ${ }^{52}$. Shortly afterwards, CLCN5 (encoding the chloride channel; CLC-5) was suggested as the candidate gene ${ }^{53}$. CIC-5 is found in lysosomes involved in endocytosis of proximal tubule proteins. Defects in the channel impair trafficking of the lysosomes to the apical membrane, leading to failure of the normal function of endocytosis; leading to the generalized failure in the re-absorptive function of the proximal tubule. The cause of the hypercalciuria seen in the condition has not been clearly delineated. It has been postulated that excess loss of phosphate and vitamin D binding protein in the urine leads to secondary increased vitamin D3 synthesis $^{54}$. Another proposed mechanism is that decreased chloride reabsorption in the proximal tubule leads to reduced calcium absorption in downstream nephron segments (presumably by reducing the trans-tubular electrochemical gradient necessary for cation transport ${ }^{55}$ ). Nephrocalcinosis may occur in Dent patients without hypercalciuria ${ }^{56}$ and there is some evidence that defective CIC-5 in collecting duct cells may cause decreased clearing of calcium crystals from the apical cell surface ${ }^{57}$. Also, decreased proximal tubular endocytosis of parathyroid hormone (PTH) results in an increased luminal concentration of $\mathrm{PTH}$, which stimulates increased 1-alfa hydroxylation and causes decreased expression of $\mathrm{NaPi} 2$ at the apical membrane and phosphaturia, favouring calcification ${ }^{58}$. About a third of 
patients do not have a defect in CLCN5, but have a mutation in the gene $O C R L^{59}$. This is also on the $\mathrm{X}$-chromosome and encodes phosphatidylinositol 4,5-bisphosphate 5-phosphate, a protein that sits on the Golgi apparatus and directs proteins to the appropriate membrane. Again, it is thought that mutations in this gene lead to impaired trafficking of endosomes to the apical membrane. Dent patients with this mutation are sometimes referred to as Dent disease type 2. A related condition, Lowe syndrome is also caused by OCRL mutations; these patients have ocular defects and learning difficulties, along with renal Fanconi syndrome ${ }^{59}$. Patients with type 2 Dent disease and those with Lowe syndrome may actually form a spectrum of disease, rather than being phenotypically distinct cohorts.

\section{Cystinuria}

Cystinuria is characterized by the failure to reabsorb the amino acids cysteine, lysine, ornithine and arginine. The resulting concentration of cysteine in the urine is high enough to result in cystine (the oxidised dimer of cysteine) formation, which is highly insoluble, and its precipitation causing cystine nephrolithiasis. Cystinuria type $\mathrm{A}$ is due to mutations in SCL3A1, while cystinuria type $\mathrm{B}$ is due to mutations in SCL7A9, which encode the heavy $\mathrm{t}$ (rBAT) and light $x\left(b^{0,+} A T\right)$ subunits, respectively, of the renal amino acid transporter $b^{0+, 60,61}$. The heavy subunit facilitates the localisation of the channel to the apical membrane in proximal tubule epithelial cells. The light subunit carries out the catalytic functions of the transporter. $b^{0+}$ transports cysteine and dibasic amino acids in exchange for neutral amino acids ${ }^{62}$. Not surprisingly, SCL3A1 mutations lead to trafficking defects, while SCL7A9 
mutations lead to loss of function of the exchanger. Although this condition leads to failure to reabsorb cysteine, lysine, ornithine and arginine, it is cysteine that causes the clinical problem due to its very low solubility in urine at neutral and acidic $\mathrm{pH}^{63}$.

\section{Conclusions}

The proximal tubule has an important role to play in maintaining many normal physiological processes in the body. While the distal nephron segments can fine-tune the final composition of urine, the heavy work, at least in relation to solute reclamation, has already occurred in the proximal tubule. Of all the processes occurring in the immediate post-glomerular nephron, it is abnormalities of phosphate reabsorption that are more commonly implicated in nephrolithiasis. The first transporter in this segment to be associated with nephrolithiasis was $\mathrm{NaPi}-\mathrm{Ilc}$, despite it only transporting a fraction of the phosphate transported by $\mathrm{NaPi}$-Ila. For a long time, no convincing evidence could be found linking $\mathrm{NaPi}$-lla to human disease. However, in the last ten years has seen a number of mutations linked to dysfunctional $\mathrm{NaPi}-\mathrm{Ila}$ and nephrolithiasis. However, there is marked phenotypic heterogeneity seen with mutations in these transporters, from an isolated phosphate leak to a more 
generalized proximal tubulopathy, and even a full-blown Fanconi syndrome. Uncovering what lies behind these differences will lead to a better understanding of the factors governing stone risk attributable to altered proximal tubule function. 


\section{References}

1. Curthoys, N. P. \& Moe, O. W. Proximal Tubule Function and Response to Acidosis. Clin. J. Am. Soc. Nephrol. 9, 1627-1638 (2014).

2. Jones, G., Prosser, D. E. \& Kaufmann, M. 25-Hydroxyvitamin D-24-hydroxylase (CYP24A1): Its important role in the degradation of vitamin D. Arch. Biochem. Biophys. 523, 9-18 (2012).

3. Wagner, C. A., Hernando, N., Forster, I. C. \& Biber, J. The SLC34 family of sodium-dependent phosphate transporters. Pflugers Arch. 466, 139-153 (2014).

4. Villa-Bellosta, R. et al. The Na+-Pi cotransporter PiT-2 (SLC20A2) is expressed in the apical membrane of rat renal proximal tubules and regulated by dietary Pi. Am. J. Physiol. Renal Physiol. 296, F691-699 (2009).

5. Ullrich, K. J. \& Murer, H. Sulphate and phosphate transport in the renal proximal tubule. Philos. Trans. R. Soc. Lond. B. Biol. Sci. 299, 549-558 (1982).

6. Offermanns, S., Iida-Klein, A., Segre, G. V. \& Simon, M. I. G alpha q family members couple parathyroid hormone (PTH)/PTH-related peptide and calcitonin receptors to phospholipase C in COS-7 cells. Mol. Endocrinol. (2009). doi:10.1210/mend.10.5.8732687

7. Abou-Samra, A. B. et al. Expression cloning of a common receptor for parathyroid hormone and parathyroid hormone-related peptide from rat osteoblast-like cells: a single receptor stimulates intracellular accumulation of both cAMP and inositol trisphosphates and increases intracellular free calcium. Proc. Natl. Acad. Sci. 89, 2732-2736 (1992). 
8. Mahon, M. J., Donowitz, M., Yun, C. C. \& Segre, G. V. Na(+)/H(+ ) exchanger regulatory factor 2 directs parathyroid hormone 1 receptor signalling. Nature 417, 858-861 (2002).

9. Gisler, S. M. et al. Interaction of the type IIa Na/Pi cotransporter with PDZ proteins. J. Biol. Chem. 276, 9206-9213 (2001).

10. Murer, H., Hernando, N., Forster, I. \& Biber, J. Proximal Tubular Phosphate Reabsorption: Molecular Mechanisms. Physiol. Rev. 80, 1373-1409 (2000).

11. Bacic, D. et al. Involvement of the MAPK-kinase pathway in the PTHmediated regulation of the proximal tubule type IIa Na+/Pi cotransporter in mouse kidney. Pflugers Arch. 446, 52-60 (2003).

12. Collazo, R. et al. Acute regulation of $\mathrm{Na}+/ \mathrm{H}+$ exchanger NHE3 by parathyroid hormone via NHE3 phosphorylation and dynamin-dependent endocytosis. J. Biol. Chem. 275, 31601-31608 (2000).

13. Schnermann, J., Huang, Y. \& Mizel, D. Fluid reabsorption in proximal convoluted tubules of mice with gene deletions of claudin- 2 and/or aquaporin1. Am. J. Physiol. Renal Physiol. 305, F1352-1364 (2013).

14. Agus, Z. S., Gardner, L. B., Beck, L. H. \& Goldberg, M. Effects of parathyroid hormone on renal tubular reabsorption of calcium, sodium, and phosphate. Am. J. Physiol. 224, 1143-1148 (1973).

15. Edwards, B. R., Baer, P. G., Sutton, R. A. L. \& Dirks, J. H. Micropuncture Study of Diuretic Effects on Sodium and Calcium Reabsorption in the Dog Nephron. J. Clin. Invest. 52, 2418-2427 (1973).

16. Pan, W. et al. The epithelial sodium/proton exchanger, NHE3, is necessary for renal and intestinal calcium (re)absorption. Am. J. Physiol.-Ren. Physiol. 302, F943-F956 (2011). 
17. Capasso, G. et al. The calcium sensing receptor modulates fluid reabsorption and acid secretion in the proximal tubule. Kidney Int. 84, 277284 (2013).

18. Ba, J., Brown, D. \& Friedman, P. A. Calcium-sensing receptor regulation of PTH-inhibitable proximal tubule phosphate transport. Am. J. Physiol.-Ren. Physiol. 285, F1233-F1243 (2003).

19. Riccardi, D. et al. Localization of the extracellular $\mathrm{Ca}(2+)$-sensing receptor and PTH/PTHrP receptor in rat kidney. Am. J. Physiol. 271, F951-956 (1996).

20. Riccardi, D. et al. Localization of the extracellular Ca2+/polyvalent cationsensing protein in rat kidney. Am. J. Physiol. 274, F611-622 (1998).

21. Loupy, A. et al. PTH-independent regulation of blood calcium concentration by the calcium-sensing receptor. J. Clin. Invest. 122, 3355-3367 (2012).

22. Graca, J. a. Z. et al. Comparative expression of the extracellular calciumsensing receptor in the mouse, rat, and human kidney. Am. J. Physiol. Renal Physiol. 310, F518-533 (2016).

23. Farrow, E. G., Davis, S. I., Summers, L. J. \& White, K. E. Initial FGF23mediated signaling occurs in the distal convoluted tubule. J. Am. Soc. Nephrol. JASN 20, 955-960 (2009).

24. Shimada, T. et al. Vitamin D receptor-independent FGF23 actions in regulating phosphate and vitamin D metabolism. Am. J. Physiol. Renal Physiol. 289, F1088-1095 (2005).

25. Hartmann, C. M. et al. Structure of murine and human renal type II Na+phosphate cotransporter genes (Npt2 and NPT2). Proc. Natl. Acad. Sci. U. S. A. 93, 7409-7414 (1996). 
26. Kos, C. H. et al. Localization of a renal sodium-phosphate cotransporter gene to human chromosome 5q35. Genomics 19, 176-177 (1994).

27. Magen, D. et al. A loss-of-function mutation in NaPi-IIa and renal Fanconi's syndrome. N. Engl. J. Med. 362, 1102-1109 (2010).

28. Schlingmann, K. P. et al. Autosomal-Recessive Mutations in SLC34A1 Encoding Sodium-Phosphate Cotransporter 2A Cause Idiopathic Infantile Hypercalcemia. J. Am. Soc. Nephrol. JASN 27, 604-614 (2016).

29. Demir, K. et al. Clinical Heterogeneity and Phenotypic Expansion of NaPiIIa-Associated Disease. J. Clin. Endocrinol. Metab. 102, 4604-4614 (2017).

30. Prié, D. et al. Nephrolithiasis and Osteoporosis Associated with Hypophosphatemia Caused by Mutations in the Type 2a Sodium-Phosphate Cotransporter. N. Engl. J. Med. 347, 983-991 (2002).

31. Virkki Leila V, Forster Ian C, Hernando Nati, Biber JÜRg \& Murer Heini. Functional Characterization of Two Naturally Occurring Mutations in the Human Sodium - Phosphate Cotransporter Type IIa. J. Bone Miner. Res. 18, 2135-2141 (2009).

32. Capuano, P. et al. Defective coupling of apical PTH receptors to phospholipase $\mathrm{C}$ prevents internalization of the $\mathrm{Na}+-$ phosphate cotransporter NaPi-IIa in Nherf1-deficient mice. Am. J. Physiol.-Cell Physiol. 292, C927-C934 (2007).

33. Karim, Z. et al. NHERF1 mutations and responsiveness of renal parathyroid hormone. N. Engl. J. Med. 359, 1128-1135 (2008). 
34. Courbebaisse, M. et al. A New Human NHERF1 Mutation Decreases Renal Phosphate Transporter NPT2a Expression by a PTH-Independent Mechanism. PLoS ONE 7, (2012).

35. Urabe, Y. et al. A Genome-Wide Association Study of Nephrolithiasis in the Japanese Population Identifies Novel Susceptible Loci at 5q35.3, 7p14.3, and 13q14.1. PLoS Genet. 8, (2012).

36. Kestenbaum, B. et al. Common genetic variants associate with serum phosphorus concentration. J. Am. Soc. Nephrol. JASN 21, 1223-1232 (2010).

37. Beck, L. et al. Targeted inactivation of Npt2 in mice leads to severe renal phosphate wasting, hypercalciuria, and skeletal abnormalities. Proc. Natl. Acad. Sci. U. S. A. 95, 5372-5377 (1998).

38. Lorenz-Depiereux, B. et al. Hereditary hypophosphatemic rickets with hypercalciuria is caused by mutations in the sodium-phosphate cotransporter gene SLC34A3. Am. J. Hum. Genet. 78, 193-201 (2006).

39. Bergwitz, C. et al. SLC34A3 Mutations in Patients with Hereditary Hypophosphatemic Rickets with Hypercalciuria Predict a Key Role for the Sodium-Phosphate Cotransporter NaPi-IIc in Maintaining Phosphate Homeostasis. Am. J. Hum. Genet. 78, 179-192 (2006).

40. Tieder, M. et al. Hereditary hypophosphatemic rickets with hypercalciuria. N. Engl. J. Med. 312, 611-617 (1985).

41. Phulwani, P., Bergwitz, C., Jaureguiberry, G., Rasoulpour, M. \& Estrada, E. Hereditary Hypophosphatemic Rickets With Hypercalciuria and Nephrolithiasis-Identification of a Novel SLC34A3/NaPi-IIc Mutation. Am. J. Med. Genet. A. 0, 626-633 (2011). 
42. Wolf, M. T. F. et al. Mapping a new suggestive gene locus for autosomal dominant nephrolithiasis to chromosome 9q33.2-q34.2 by total genome search for linkage. Nephrol. Dial. Transplant. 20, 909-914 (2005).

43. Dasgupta, D. et al. Mutations in SLC34A3/NPT2c Are Associated with Kidney Stones and Nephrocalcinosis. J. Am. Soc. Nephrol. 25, 2366-2375 (2014).

44. Schlingmann, K. P. et al. Mutations in CYP24A1 and idiopathic infantile hypercalcemia. N. Engl. J. Med. 365, 410-421 (2011).

45. Nesterova, G. et al. 1,25-(OH)2D-24 Hydroxylase (CYP24A1) Deficiency as a Cause of Nephrolithiasis. Clin. J. Am. Soc. Nephrol. CJASN 8, 649-657 (2013).

46. Vezzoli, G., Terranegra, A. \& Soldati, L. Calcium-sensing receptor gene polymorphisms in patients with calcium nephrolithiasis. Curr. Opin. Nephrol. Hypertens. 21, 355-361 (2012).

47. Vezzoli, G. et al. R990G polymorphism of calcium-sensing receptor does produce a gain-of-function and predispose to primary hypercalciuria. Kidney Int. 71, 1155-1162 (2007).

48. Harding, B. et al. Functional characterization of calcium sensing receptor polymorphisms and absence of association with indices of calcium homeostasis and bone mineral density. Clin. Endocrinol. (Oxf.) 65, 598-605 (2006).

49. Vezzoli, G. et al. Risk of nephrolithiasis in primary hyperparathyroidism is associated with two polymorphisms of the calcium-sensing receptor gene. J. Nephrol. 28, 67-72 (2015).

50. Dent, C. E. \& Friedman, M. Hypercalcuric Rickets Associated with Renal Tubular Damage. Arch. Dis. Child. 39, 240-249 (1964). 
51. Wrong, O. M., Norden, A. G. W. \& Feest, T. G. Dent's disease; a familial proximal renal tubular syndrome with low-molecular-weight proteinuria, hypercalciuria, nephrocalcinosis, metabolic bone disease, progressive renal failure and a marked male predominance. QJM 87, 473-493 (1994).

52. Scheinman, S. J. et al. Mapping the gene causing X-linked recessive nephrolithiasis to Xp11.22 by linkage studies. J. Clin. Invest. 91, 2351-2357 (1993).

53. Lloyd, S. E. et al. A common molecular basis for three inherited kidney stone diseases. Nature 379, 445-449 (1996).

54. Devuyst, O. \& Thakker, R. V. Dent's disease. Orphanet J. Rare Dis. 5, 28 (2010).

55. Unwin, R., Fine, L., Cohen, E., Thakker, R. \& Tanner, M. Unravelling of the molecular mechanisms of kidney stones. The Lancet 348, 1561-1565 (1996).

56. Ludwig, M. et al. Hypercalciuria in patients with CLCN5 mutations. Pediatr. Nephrol. 21, 1241-1250 (2006).

57. Sayer, J. A., Carr, G. \& Simmons, N. L. Calcium phosphate and calcium oxalate crystal handling is dependent upon CLC-5 expression in mouse collecting duct cells. Biochim. Biophys. Acta BBA - Mol. Basis Dis. 1689, 83-90 (2004).

58. Piwon, N., Günther, W., Schwake, M., Bösl, M. R. \& Jentsch, T. J. ClC-5 Cl-channel disruption impairs endocytosis in a mouse model for Dent's disease. Nature 408, 369-373 (2000).

59. Hoopes Jr., R. R. et al. Dent Disease with Mutations in OCRL1. Am. J. Hum. Genet. 76, 260-267 (2005). 
60. Calonge, M. J. et al. Cystinuria caused by mutations in rBAT, a gene involved in the transport of cystine. Nat. Genet. 6, 420-425 (1994).

61. Feliubadaló, L. et al. Non-type I cystinuria caused by mutations in SLC7A9, encoding a subunit (bo,+AT) of rBAT. Nat. Genet. 23, 52-57 (1999).

62. Chillarón, J. et al. Pathophysiology and treatment of cystinuria. Nat. Rev. Nephrol. 6, 424-434 (2010).

63. Bowden, N. A., Sanders, J. P. M. \& Bruins, M. E. Solubility of the Proteinogenic $\alpha$-Amino Acids in Water, Ethanol, and Ethanol-Water Mixtures. J. Chem. Eng. Data (2018). doi:10.1021/acs.jced.7b00486

\section{Figure 1}

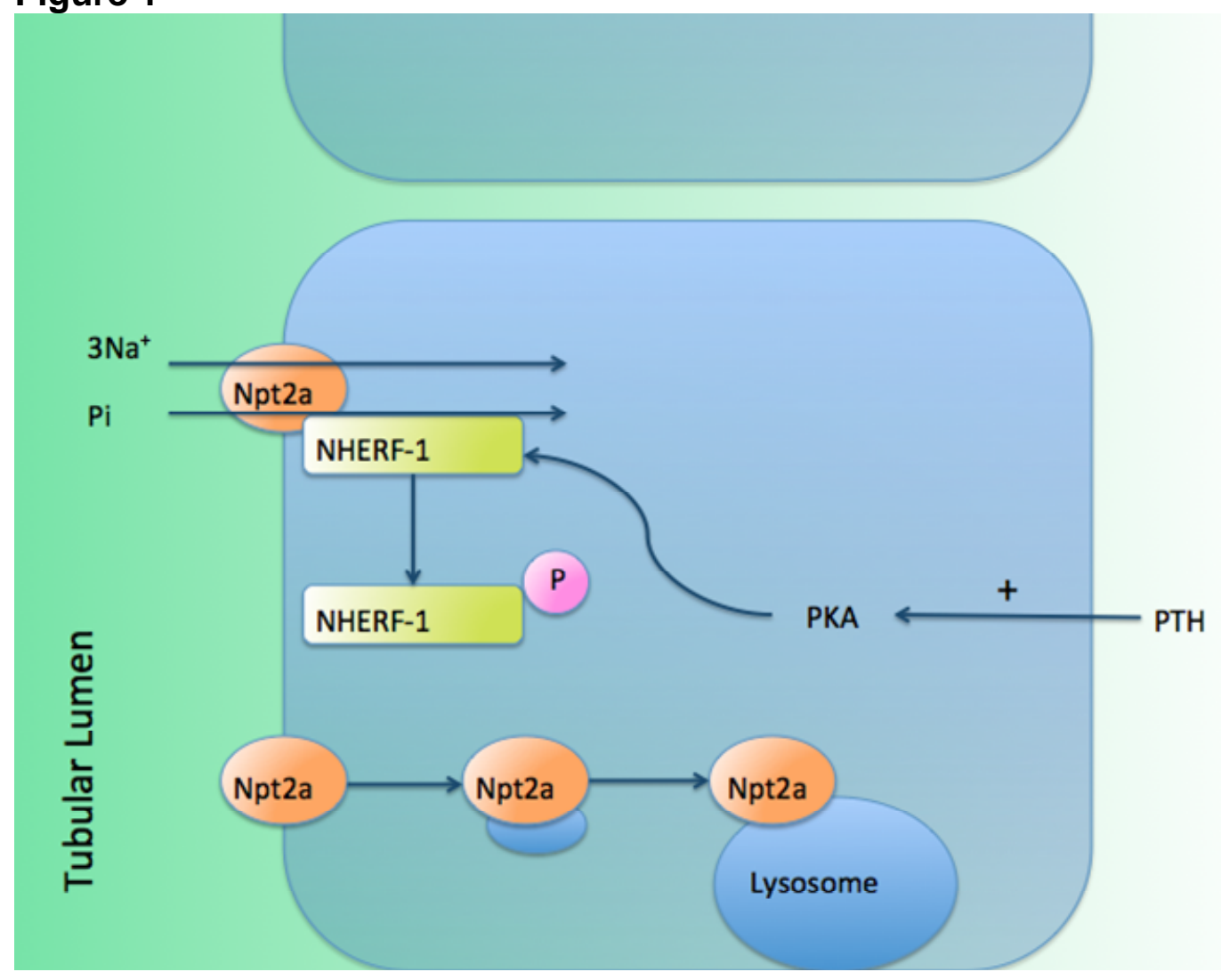

\section{Figure Legends}

\section{Figure 1}

Proximal tubular cell showing the sodium/phosphate cotransporter NaPi-Ila. The scaffolding protein NHERF-1 holds NaPi-lla to the apical membrane facilitating sodium and phosphate reabsorption. The action of PTH activates 
protein kinase $A(P K A)$, which in turn phosphorylates NHERF-1.

Phosphorylated NHERF-1 no longer anchors NaPi-lla, leaving it free to be endocytosed in lysosomes preventing phosphate and sodium reabsorption. 